\title{
Assessment of the percentage of apparent diffusion coefficient value changes as an early indicator of the response of colorectal hepatic metastases to chemotherapy
}

\author{
Nada Gamal El-Husseiny', Sayed Mohamed Mehana ${ }^{1 *}$ (1) and Sherif Farouk El Zawawy ${ }^{2}$
}

\begin{abstract}
Background: Colorectal cancer is considered one of the most common causes of cancer-related deaths worldwide. We aim to evaluate the efficacy of DWI-MRI in predicting response to chemotherapy in this cohort.

The study included 30 lesions in 20 biopsy proven-colorectal cancer patients with hepatic metastasis larger than 1 $\mathrm{cm}$. All patients underwent both triphasic CT with intravenous contrast, pre-chemotherapy MRI (axial T2 and DW sequences) which was repeated 21 days following chemotherapy. A follow-up CT was done 2 months later. The response of the lesions was evaluated using the RESCIST criteria. On MRI, the lesions corresponding to the ones chosen on CT were identified and the apparent diffusion coefficient (ADC) values of pre- and post-chemotherapy images were recorded and correlated with the CT results.

Results: In the study, 17 (56.7\%) of the lesions showed response to chemotherapy while 13 (43.3\%) were nonresponding. There was no significant difference in pretreatment ADC values between responding and nonresponding lesions $(p=0.14)$. The mean percentage increase in ADC values in responding lesions was $42 \%$ compared to $18 \%$ in non-responding lesions $(p<0.001)$. Lesions that showed less than $18 \%$ increase were all found to be non-responsive

Conclusion: DWI-MRI has an emerging role in early assessment of early treatment response that can be detected before morphological response for patients with hepatic metastasis from colorectal cancer. Based on our study, the use of $25 \%$ as the cutoff point of percent difference in ADC for detection of non-responding lesions proved to be successful only 21 days after the 1st chemotherapy cycle.
\end{abstract}

Keywords: Magnetic resonance imaging, Diffusion magnetic resonance imaging, Colorectal neoplasms, Response evaluation criteria in solid tumors, Follow-up studies

\section{Key points}

Evaluation of the efficacy of DWI-MRI in predicting response to chemotherapy

Apparent diffusion coefficient (ADC) values of pre- and post-chemotherapy images for hepatic metastases from colorectal cancers

The cutoff points of percent difference in ADC for detection of non-responding hepatic metastases

\footnotetext{
* Correspondence: sayedmehana9@gmail.com

'Department of Diagnostic and Intervention Radiodiagnosis, Medical Research Institute, Alexandria University, 165 Alhoreia Street, Alexandria 21561, Egypt

Full list of author information is available at the end of the article
}

\section{Background}

Colorectal carcinoma (CRC) is considered the third most common cancer in males and the second in females worldwide, it is responsible for over $9 \%$ of all cancer incidence [1]. Around $60 \%$ of patients with colorectal carcinoma develop a lymph-nodal or a distant metastasis, the liver as the initial site in $30 \%$ of hematogenous metastasis [2].

According to the Response Evaluation Criteria in Solid Tumors (RECIST) version 1.1, the size change is currently considered the bases of evaluation of therapeutic response after chemotherapy [3]. However, because 
therapy may result in modification in tissue composition despite constant tumor size; moreover, fatty liver infiltration after chemotherapy can affect the liver parenchyma impairing the assessment of lesions, functional techniques, such as dynamic contrast-enhanced magnetic resonance imaging (DCE-MRI) and diffusion-weighted imaging (DWI), which reveal alterations in tissue microstructure rather than macrostructure changes $[4,5]$.

Apparent diffusion coefficient (ADC) depends on the tissue cellularity. Malignant lesions, such as liver metastases frequently demonstrate low ADC, except in treated lesions or internal necrosis [6]. Tumors with low ADC values before the beginning of the treatment that significantly increased following the first cycle of chemotherapy are suggested in cases of good therapeutic response with a greater decrease in tumor volume after chemotherapy $[7,8]$.

Despite being not considered in RECIST 1.1, recent publications considered diffusion-weighted MRI (DWMRI) a promising technique in cancer patients and tumoral response [9]. However, studies on its use for prediction of the response of colorectal liver metastases are

Table 1 Site, number, and size of liver lesions

\begin{tabular}{|c|c|c|c|}
\hline \multicolumn{2}{|l|}{ Variable } & No. of patients & (\%) \\
\hline \multicolumn{4}{|c|}{ Location of liver deposits } \\
\hline \multicolumn{2}{|l|}{ Segment III } & 4 & 13.3 \\
\hline \multicolumn{2}{|l|}{ Segment IV } & 6 & 20.0 \\
\hline \multicolumn{2}{|l|}{ Segment V } & 3 & 10 \\
\hline \multicolumn{2}{|l|}{ Segment Vl } & 1 & 3.3 \\
\hline \multicolumn{2}{|l|}{ Segment VII } & 8 & 26.7 \\
\hline \multicolumn{2}{|l|}{ Segment VIII } & 6 & 20.0 \\
\hline \multicolumn{2}{|c|}{ Segment VIIIN interface } & 1 & 3.3 \\
\hline \multicolumn{2}{|c|}{ Segment VII/VIII interface } & 1 & 3.3 \\
\hline \multicolumn{4}{|c|}{ Initial number of lesions } \\
\hline \multicolumn{2}{|l|}{1} & 2 & 10.0 \\
\hline \multicolumn{2}{|l|}{2} & 2 & 10.0 \\
\hline \multicolumn{2}{|l|}{3} & 2 & 10.0 \\
\hline \multicolumn{2}{|l|}{4} & 2 & 10.0 \\
\hline \multicolumn{2}{|l|}{6} & 1 & 5.0 \\
\hline \multicolumn{2}{|l|}{ Multiple (> 6) } & 11 & 55.0 \\
\hline Lesion size & Median & Mean \pm SD & $p$ value \\
\hline \multicolumn{3}{|c|}{ MRI (cm) after 21 days } & \multirow[t]{3}{*}{0.056} \\
\hline Pre-treatment & 3.10 & $4.05 \pm 2.73$ & \\
\hline Post-treatment & 2.80 & $3.84 \pm 2.78$ & \\
\hline \multicolumn{2}{|c|}{$\mathrm{CT}(\mathrm{cm})$ after 2 months } & & \multirow[t]{3}{*}{$0.001^{*}$} \\
\hline Pre-treatment & 2.98 & $3.87 \pm 2.80$ & \\
\hline Post-treatment & 2.07 & $2.75 \pm 2.38$ & \\
\hline
\end{tabular}

limited. So, we aim to investigate the efficacy of DWIMRI in predicting the response of liver metastases from colorectal cancer to systemic treatment.

\section{Methods}

This is a prospective study of 20 colorectal cancer patients with liver metastases

\section{Inclusion criteria}

Patients having hepatic metastases from pathologically proven colorectal origin with a minimum of one liver metastasis measuring at least $1 \mathrm{~cm}$

\section{Exclusion criteria}

History of any other malignant disease, pregnancy, contraindications to MR imaging, and patient with contraindications to chemotherapy

\section{Imaging}

Eighteen patients performed pre-chemotherapy baseline triphasic CT with contrast. In the two remaining patients, contrast was not given due to allergy and elevated renal function, and diagnosis of metastases was based on their new appearance compared with the older studies of the patients in US and non-contrast CT while assessment of the size in the current study was done in the T2-weighted MRI sequence.

Lesions were evaluated at the portal phase, and the selected lesion/s was the largest with the maximum number of two lesions chosen as target lesions.

Baseline pre-chemotherapy axial T2-weighted sequence and DW MRI were then done before the first cycle of chemotherapy and repeated 21 days later just before the second cycle.

A follow-up CT was done 2 months later with the measurement of the target lesions.

CT examinations were performed using 64 slice equipment (acquiring images from the top of the liver to the pubic symphysis, before and after the intravenous injection of contrast agent (1.5 ml/kg body weight), during arterial and portal venous phases (35-40 s and 70-75 s after injection, respectively).

MRI was performed using a 1.5-T MRI scanner (SIEMENS-MAGNETOM_ ESSENZA) equipped with phased-array torso surface coil.

Table 2 Descriptive analysis of the studied cases according to $\operatorname{ADC}(n=30)$

\begin{tabular}{llll}
\hline ADC & Min.-Max. & Mean \pm SD & Median \\
\hline Pre-chemo & $0.65-1.90$ & $1.07 \pm 0.30$ & 1.0 \\
Post-chemo & $0.72-1.95$ & $1.40 \pm 0.31$ & 1.41 \\
\% difference & $0.0-99.0$ & $31.67 \pm 23.80$ & 29.50 \\
\hline
\end{tabular}


Table 3 Relation between response with ADC and \% difference of size regression

\begin{tabular}{lllll}
\hline & \multicolumn{2}{l}{ Response } & Test of Sig. & $p$ \\
\cline { 2 - 3 } & $\begin{array}{l}\text { Non-responding } \\
(n=13)\end{array}$ & $\begin{array}{l}\text { Responding } \\
(n=17)\end{array}$ & & \\
\hline ADC & & & & \\
Pre-chemo & & $1.01 \pm 0.22$ & $t=1.171$ & 0.256 \\
Mean \pm SD. & $1.14 \pm 0.37$ & 1.0 & & \\
Median & 1.0 & & & \\
Post-chemo & & $1.46 \pm 0.28$ & $t=1.305$ & 0.203 \\
Mean \pm SD. & $1.32 \pm 0.33$ & 1.52 & & \\
Median & 1.30 & & & \\
\% difference & & $39.94 \pm 16.98$ & $U=17.000^{*}$ & $<0.001^{*}$ \\
Mean \pm SD. & $16.23 \pm 25.30$ & 38.0 & & \\
Median & 11.0 & 38.0 &
\end{tabular}

$\mathrm{t}, \mathrm{p}: t$ and $p$ values for Student $t$ test for comparing between the two categories

$\mathrm{U}, \mathrm{p}: \mathrm{U}$ and $p$ values for Mann-Whitney test for comparing between the two categories

*Statistically significant at $p \leq 0.05$

Axial T2-weighted free-breathing, with TR (2000), TE (80), matrix $(256 \times 179)$, slice thickness $(6.5 \mathrm{~mm})$, and gap $(20 \%=0.8 \mathrm{~mm})$ in the axial plane.

Diffusion-weighted echo-planar imaging (EPI) sequence with TR (4500), TE (73), matrix (192 × 120), slice thickness $(7 \mathrm{~mm})$, and gap $(50 \%=2 \mathrm{~mm})$ in axial plane. The $b$ values were 50, 400, and 800 with computer-generated ADC map.

\section{Image analysis on MRI}

Images were analyzed by two radiologists independently (consultant and resident), the results were compared, and mismatching results were re-assessed in conjoint.
The lesions corresponding to the ones chosen on CT were identified.

A similar-sized ROI was placed at the same site on the pre- and post-chemotherapeutic images and the two $\mathrm{ADC}$ values were compared.

\section{CT image analysis}

The maximum diameter of each metastatic lesion was measured before and after treatment. Analysis of the results was done according to the Response Evaluation Criteria in Solid Tumors (RECIST).

A metastatic lesion presenting a $20 \%$ or more increase in the maximum transverse diameter with respect to time 0 was classified as progressive disease (PD), a lesion showing at least $30 \%$ of reduction in the maximum transverse diameter with respect to time 0 was classified as partial response (PR). A lesion not presenting such dimensional decrease or increase was considered stable disease (SD). From a clinical point of view, lesions with complete cure or regressing ( $\mathrm{CR}$ and $\mathrm{PR}$, respectively) were also labeled as responding $(\mathrm{R})$, while those progressing and stable were classified as not responding (NR).

\section{Statistical analysis}

Data were fed to the computer and analyzed using IBM SPSS software package version 20.0 (Armonk, NY: IBM Corp). Qualitative data were described using number and percent. Quantitative data were described using range (minimum and maximum), mean, standard deviation, and median. Significance of the obtained results was judged at the $5 \%$ level.

\section{Results}

Our cohort consisted of 11 males and 9 females with a mean age of $57.4 \pm 11.5$ years. Two different chemotherapeutic regimens were used; XELOX (Capecitabine and

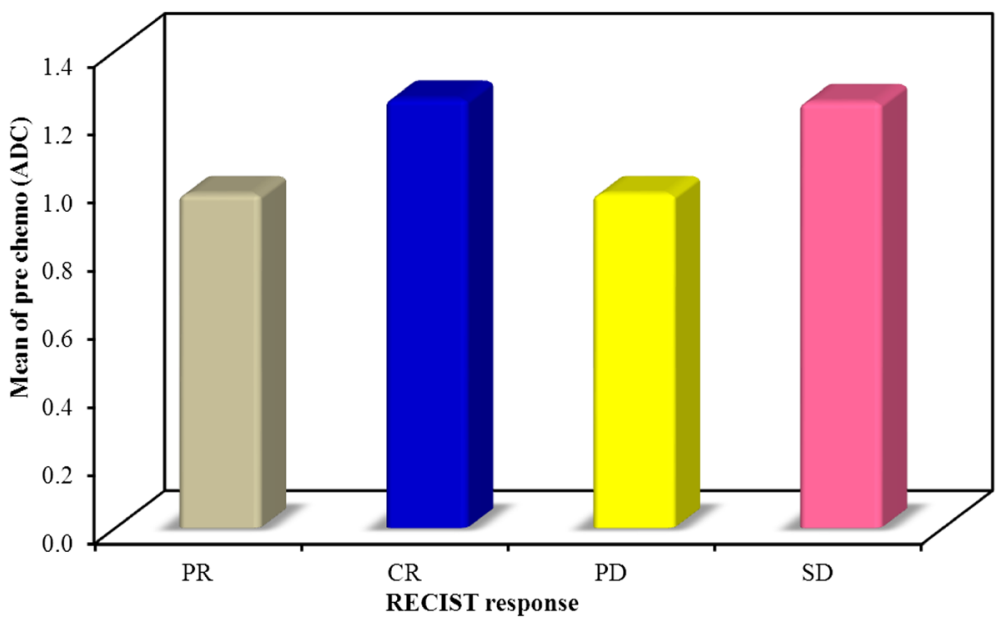

Fig. 1 Pre-treatment ADC values in different response 


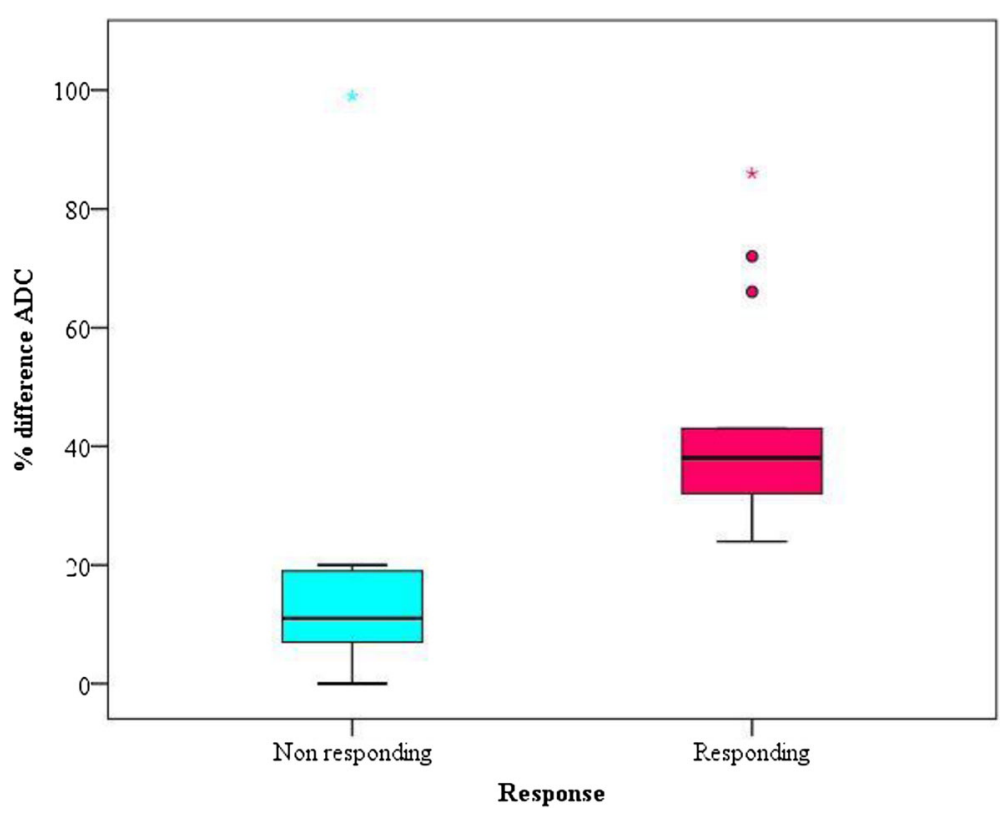

Fig. 2 Relation between response and percent difference ADC

Oxaliplatin) was used in 15/20 (75\%) patients. While FOLFOX (5FU, Leucovorin and Oxaliplatin) was used in 5/20 $(15 \%)$

Details about the number, location, and size of the lesions before and after chemotherapy are presented in Table 1.

Our data show that there is no significant morphological changes in size in the initial MRI study performed 3 weeks after initiation of the treatment with mean percentage change in the size was $5 \%(p=$ 0.056) (Table 1).

The lesion response on CT was evaluated using the RECIST criteria, 15 (50 \%) of the lesions showed PR. 4 lesions showed were completely disappeared (complete response $(\mathrm{CR})$ ), while 4 lesions showed PD. Seven lesions were characterized as stable (SD).

In view of the RECIST criteria, 19 lesions were overall classified as responding to the chemotherapy (PR and CR lesions) while 11 showed no significant response and were classified as non-responding (SD and PD).

All of the patients were subjected to diffusionweighted MRI, pre- and post-chemotherapy. The mean percentage difference was about $31.67 \%$ (Table 2). Details presented in Table 3.

There was no statistically significant difference between the mean pre-chemotherapeutic ADC values of the responding lesions (PR and $\mathrm{CR}$ ) and non-responding lesions (PD and SD) $(p=0.139)$. Therefore, in our study, the pretreatment ADC value was not a good predictor of tumor response (Fig. 1).

The mean percentage increase in ADC value seen in responding lesions was $39.8 \%$ while in non-responding lesions was only $16.2 \%$. The $p$ value was $<0.001$ (Table 3 ) which proved that there was indeed a positive relationship between the percentage increase in ADC and the response. In our study, out of the 30 lesions evaluated, lesions that showed less than $20 \%$ increase were all found to be non-responding (12 lesions) while 17 out of 18 lesions that showed more than $20 \%$ increase were responding (Fig. 2, Table 4).

According to the previously stated results, the use of $25 \%$ as the cutoff point of percent difference in ADC for detection of non-responding lesions proved to be successful with a sensitivity of $92.3 \%, 100 \%$ specificity, and

Table 4 Relation between RECIST response and mean pre-, post-chemotherapy ADC, and \% difference of ADC ( $n=30)$

\begin{tabular}{|c|c|c|c|c|c|c|}
\hline \multirow[t]{2}{*}{$A D C$} & \multicolumn{4}{|c|}{ RECIST response } & \multirow[t]{2}{*}{ Test of Sig. } & \multirow[t]{2}{*}{$p$} \\
\hline & $\mathrm{PR}(n=15)$ & $\mathrm{CR}(n=2)$ & $\mathrm{PD}(n=5)$ & $\mathrm{SD}(n=8)$ & & \\
\hline Mean pre-chemo & $0.98 \pm 0.21$ & $1.26 \pm 0.25$ & $0.98 \pm 0.33$ & $1.25 \pm 0.38$ & $F=2.001$ & 0.139 \\
\hline Mean post-chemo & $1.43 \pm 0.27$ & $1.74 \pm 0.30$ & $1.21 \pm 0.29$ & $1.39 \pm 0.36$ & $F=1.540$ & 0.228 \\
\hline Mean percentage difference & $42.40 \pm 18.07$ & $36.50 \pm 3.54$ & $25.80 \pm 39.58$ & $12.63 \pm 8.48$ & $H=16.280^{*}$ & $0.001^{*}$ \\
\hline
\end{tabular}

$F, \mathrm{p}: F$ and $p$ values for ANOVA test

$\mathrm{H}, \mathrm{p}: H$ and $p$ values for Kruskal-Wallis test

*Statistically significant at $p \leq 0.05$ 


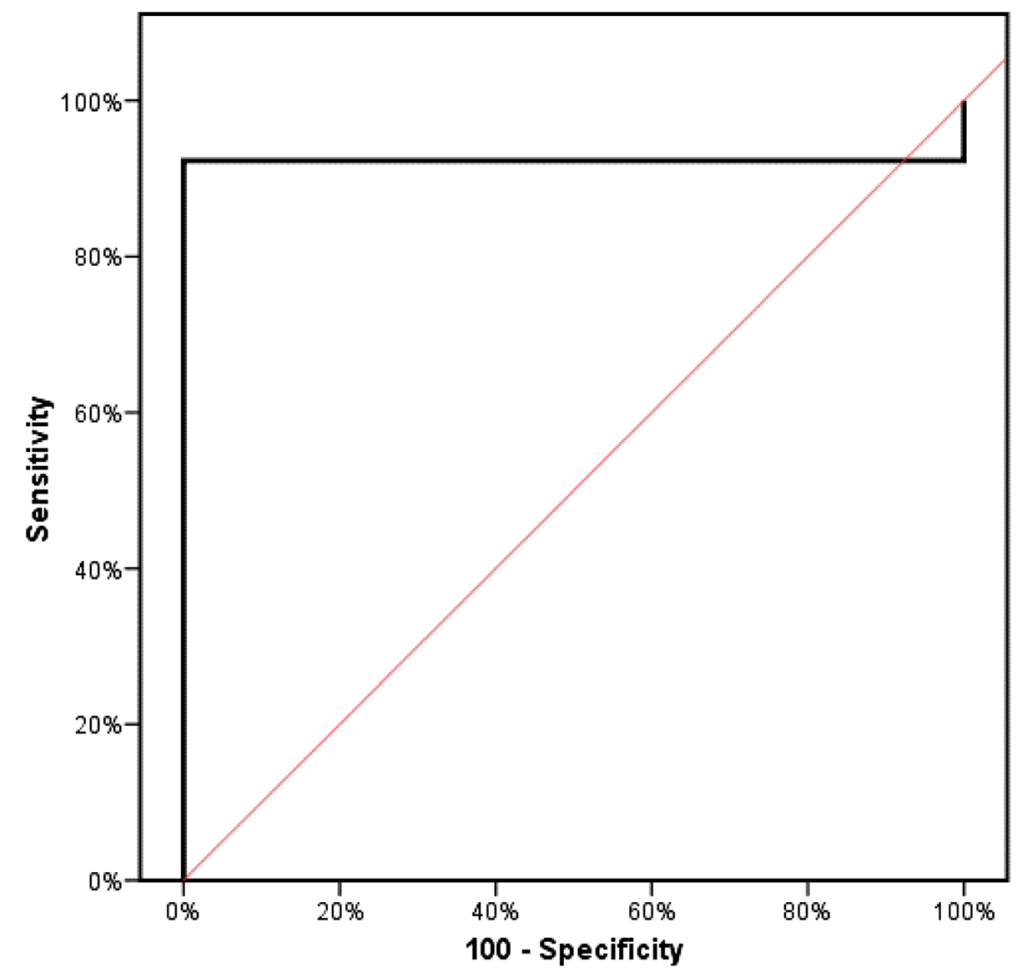

Fig. 3 ROC curve for \% difference of ADC to diagnose non-responding cases

positive predictive value of $100 \%$, and a negative predictive value was about $94 \%$ (Fig. 3).

In conclusion, our results revealed that there was no significant difference between the mean pretherapeutic ADC of the responding and nonresponding lesions. On the other hand, the responding lesions had significantly higher mean percentage difference of their ADC value showing a positive relationship between the increase in ADC and the chances of a better response.

\section{Discussion}

Tumors could respond to therapy in ways other than size reduction such as modified tissue composition which can be assessed by functional imaging such as the DWI which can detect tissue microstructure alteration rather than macrostructure [5].

Our results regarding location, number, and size of the liver lesions are all similar to the literature published about colorectal cancer with liver deposits [1].

The mean age of our cohort was 56. This is quite young compared to data from the American Cancer Society in 2017 in which the median age at diagnosis for colon cancer is 68 in men and 72 in women [10].

Regarding the size of the lesions, the mean size in a study done by Schulz et al. [11] was $1.4 \mathrm{~cm}$ compared to about $3.8 \mathrm{~cm}$ in this study which might be explained by better screening and earlier detection due to the established screening programs.

Prediction of response based on pre-treatment ADC is still controversial in some studies from the literature. Necrotic tumors are often surrounded by hypoxic viable cells that are less sensitive to ionizing radiation, more prone to aggressive behavior and probably less sensitive to cytotoxic agents [12]. Probably, these lesions would present a higher pre-treatment ADC and a worse treatment outcome [13]. Studies done by Chi et al. [14] and Kim et al. [15] confirm this theory while others like the ones done by DeVries et al. [16], Harry et al. [17] and Nilsen et al. [18] do not show significant correlation between pre-treatment ADC and response to chemo- or radiotherapy. Moreover, other studies done by $\mathrm{Kol}$ et al. [19] and Mardor et al. [20] even show negative correlations between these two variables. In our series, there was no significant difference between the ADC values between responsive and non-responsive lesions (Fig. 1) and our result cannot confirm the above hypothesis.

The second issue we tried to address in this study is that if DWI would be able to provide an early marker for treatment efficacy. Most published studies [14, 15, 21, 22] have shown that ADC changes could precede changes in tumor size; however, changes may disappear after a certain time due to repair mechanisms such as decrease of edema and organization of necrosis [23]. In a study done by Mungai et al. [24], assessment of the chemotherapeutic response 


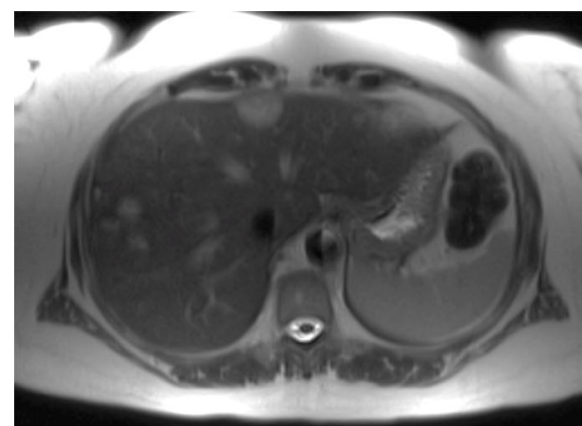

(A)

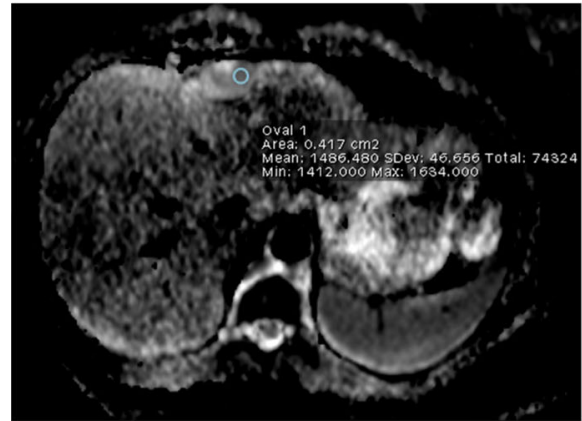

(C)

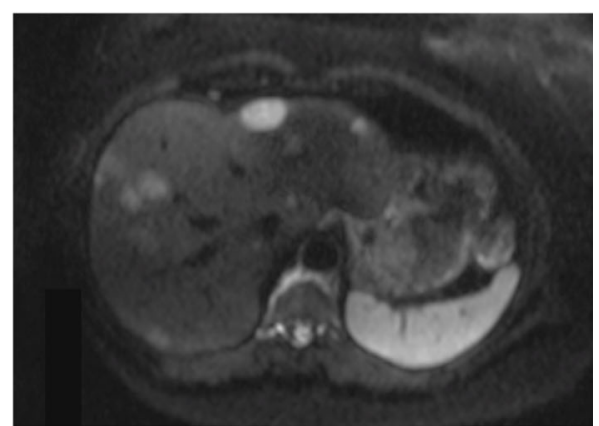

(B)

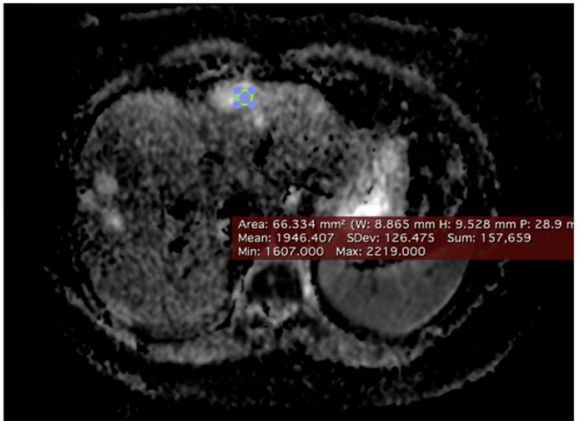

(D)

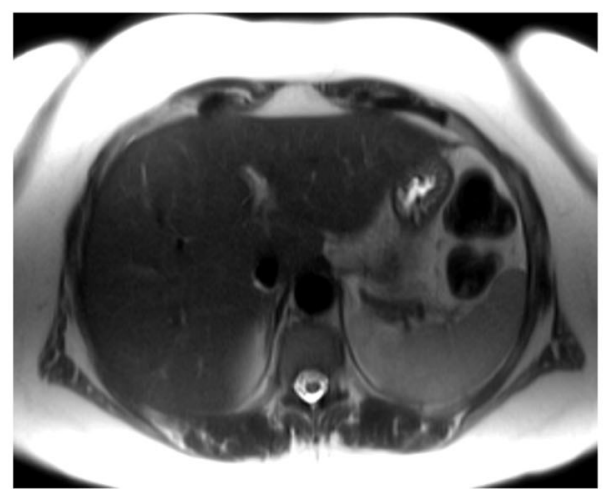

(E)

Fig. 4 Case (1): A 48 year old female patient with history of rectal adeno-carcinoma with hepatic metastasis, patient has contrast sensitivity and only non-contrast MRI was done. a) Base line pre chemotherapy axial T2 MRI revealed; multiple lesions, the target lesion is a well-defined sub capsular hyper intense lesion measuring about $2.5 \mathrm{~cm}$ seen in segment III. b) Corresponding axial baseline diffusion weighted image of the previous $T 2$ target lesion with b Value of $(800 \mathrm{~m} 2 / \mathrm{sec})$ shows the lesion displaying hyper intense signal. c) pre therapeutic ADC image revealed diffusion restriction and ADC value of $1.49 \times 10-3 \mathrm{~m} 2 / \mathrm{sec}$ (d) post therapeutic (21 days after chemotherapy) ADC map showing increase in the ADC value up to $1.96 \times 10-3 \mathrm{~m} 2 / \mathrm{sec}$ (e) 2 month follow up non contrast T2 MRI revealed total resolution of the previously noted target lesion denoting complete response according to RECIST criteria

of liver metastases appears feasible after 20-25 days since the beginning of chemotherapy.

In the current study, we followed this theory and our assessment was done 21 days after the start of chemotherapy. The post-therapeutic lesion size showed almost no change with a median size of $2.6 \mathrm{~cm}$ compared to the pre-therapeutic median of $3.0 \mathrm{~cm}$ and percentage mean change of about $5 \%$, as well as a non-significant $p$ value of 0.056 , proving that the 3 -week time period is not enough to demonstrate significant morphological changes in size. Meanwhile, the mean post-therapeutic $\mathrm{ADC}$ values of $\mathrm{R}$ lesion groups (both $\mathrm{SD}$ and $\mathrm{PR}$ ) become significantly higher showing a $39.94 \pm 16.98 \%$ increase than the corresponding pre-treatment values compared to only $16.23 \pm 25.30 \%$ increase in the mean ADC of NR lesions with a significant $p$ value of $<0.001$ (Table 4), confirming the hypothesis of reduced cellularity and increased diffusion of water molecule into the extracellular space for $\mathrm{R}$ lesions. We can say that an early (20-25 days after the beginning of the first cycle) 


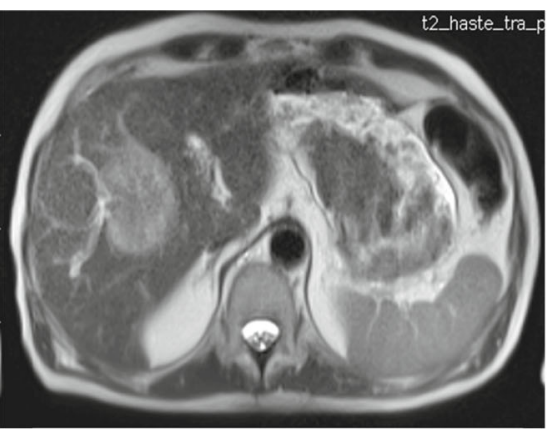

(A)

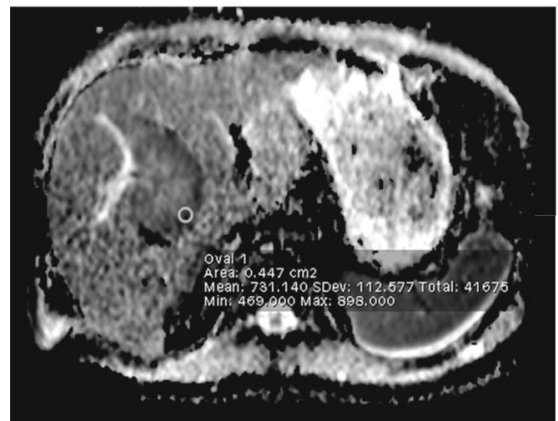

(C)

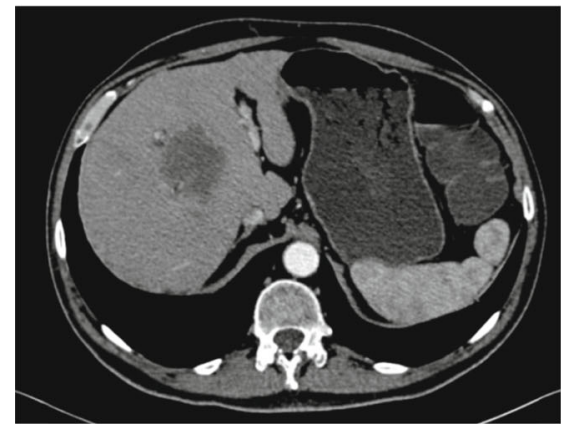

(E)

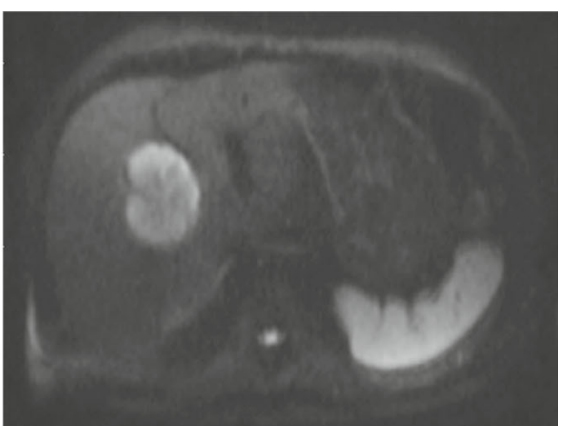

(B)

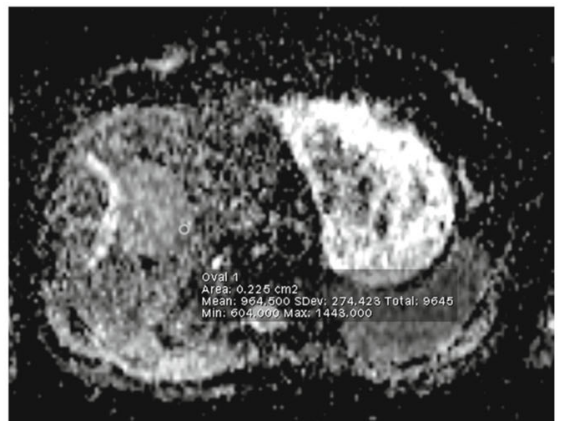

(D)

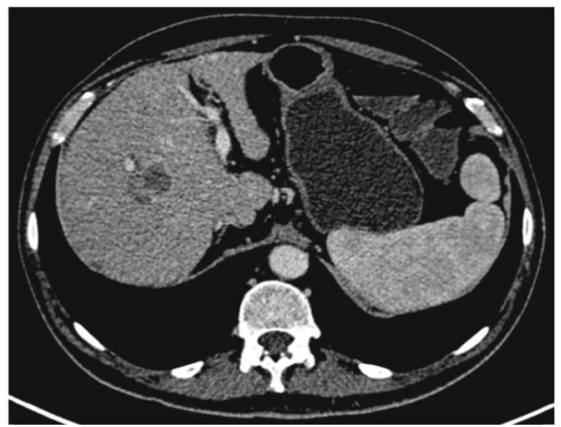

(F)

Fig. 5 Case (2): A 57 year old male patient with history of rectal adeno-carcinoma with hepatic metastasis. a) Baseline axial T2 MRI revealed mostly hyper intense lesion in segment VIIIN interface. b) Corresponding axial baseline diffusion weighted image of the previous T2 target lesion with $b$ value of $(800 \mathrm{~m} 2 / \mathrm{sec})$ shows the lesion displaying hyper intense signal. c) pre therapeutic ADC image which revealed diffusion restriction and ADC value of $0.73 \times 10-3 \mathrm{~m} 2 / \mathrm{sec}$. (d) post therapeutic (21 days after chemotherapy) ADC map showing increase in the ADC value up to 0.96 x10-3 m2/sec (e) base line pre chemotherapy CT portal phase; hypo dense lesion with relative peripheral enhancement was noted measuring about $5.6 \mathrm{~cm}$. (f) 2 month follow up CT portal phase revealed significant reduction of the lesion currently seen measuring about $2.3 \mathrm{~cm}$ (partial response according to RECIST criteria)

evaluation of ADC changes appears useful for an early assessment of clinical response.

Also in our limited study, we have reached a cutoff point of $25 \%$ which showed a very high sensitivity of $92.3 \%$ and specificity of $100 \%$ in the detection of nonresponding lesions. All of the non-responding lesions in our study showed a percentage change in their ADC value less than that cutoff point of $20 \%$ giving a positive predictive value of $100 \%$, while all of the responding lesions apart from one showed a percentage change of
ADC more than the cutoff value resulting in a negative predictive value of about $94 \%$.

However, obtaining reproducible ADC measurements in liver metastases undergoing CHT is not an easy process and many possible confounding factors have to be considered. Among these factors are location of the lesion (in particular, when a lesion is located on or near organs subject to respiratory or other kinds of movement), its histotype, the presence of internal necrotic areas $\mathrm{CHT}$, and others related to the MR-DWI 


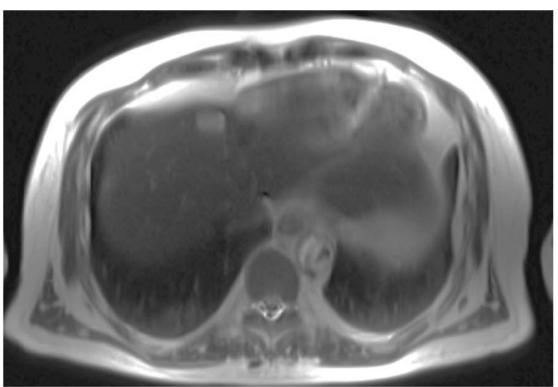

(A)

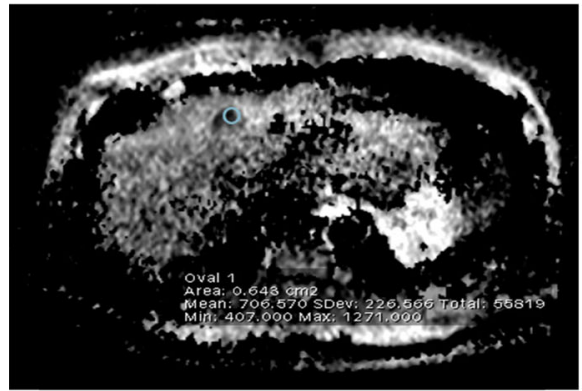

(C)

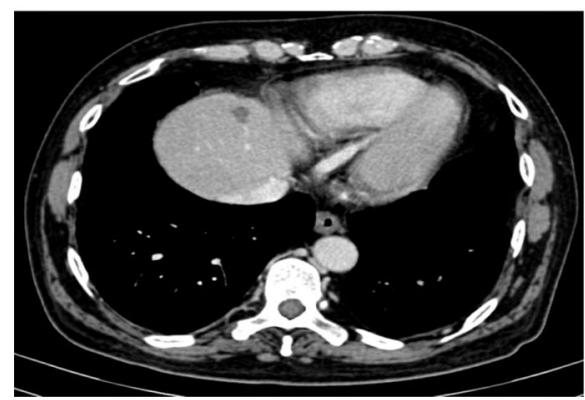

(E)

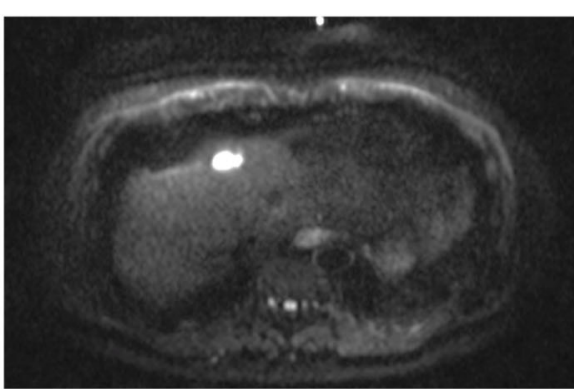

(B)

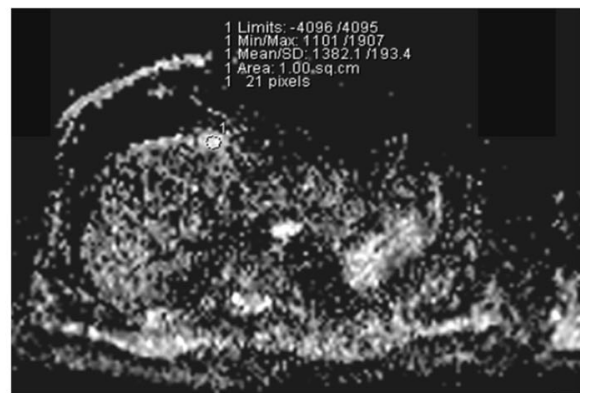

(D)

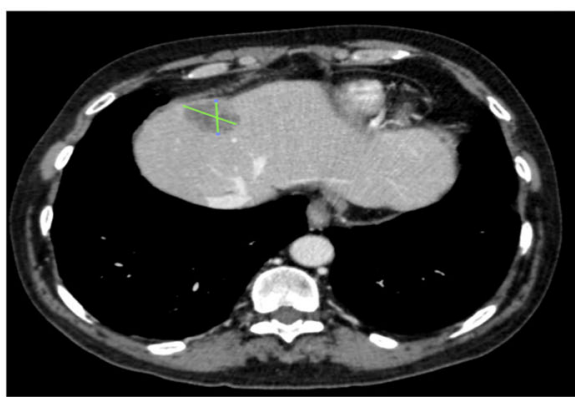

(F)

Fig. 6 Case (3): A 69 year old male patient with history of rectal adeno-carcinoma with solitary hepatic metastasis a) Baseline T2 axial MRI revealed a small solitary hepatic dome hyper intense lesion at segment IV A b) corresponding axial baseline diffusion weighted image of the previous T2 target lesion with $\mathrm{b}$ value of $(800 \mathrm{~m} 2 / \mathrm{sec})$ shows the lesion displaying hyper intense signal. c) pre therapeutic ADC image revealed diffusion restriction and ADC value of $0.71 \times 10-3 \mathrm{~m} 2 / \mathrm{sec}$. (d) post therapeutic (21 days after chemotherapy) ADC map showing marked increase in the ADC value measuring $1.38 \times 10-3 \mathrm{~m} 2 / \mathrm{sec}$ (e) base line pre chemotherapy CT portal phase; shows the same small hypo dense target lesion measuring about $1.4 \mathrm{~cm}$. (f) 2 month follow up CT portal phase revealed significant increase in the size of the lesion which was seen measuring about $3.2 \mathrm{~cm}$ (progressive disease according to RECIST criteria)

acquisition technique and ADC calculation (in particular, images low signal-to-noise ratio, acquisition artifacts, $b$ value adopted, and lesion sampling).

The current study included a small number of patients who received two-variable chemotherapy regimens, and although no significant relationship between the type of chemotherapeutic regimen used and the response obtained further studies with a larger population and more study sample homogeneity are needed. Also ideally to trace the diffusion or dynamic changes within tumor cells after neoadjuvant treatment, a functional map is required to detect changes within the same voxel at different examinations (Figs. 4, 5 and 6).

\section{Conclusion}

We believe that DWI-MRI has an emerging role in early assessment of early treatment response assessment that can be detected before morphological response on CT or conventional MRI for patients with hepatic metastasis from colorectal cancer.

Based on our study on 30 lesions, the use of $25 \%$ as the cutoff point of percent difference in ADC for detection of non-responding lesions proved to be successful with a sensitivity of $92.3 \%, 100 \%$ specificity, positive predictive value of $100 \%$, and a negative predictive value was about $94 \%$ only 21 days after the 1 st chemotherapy cycle. 


\section{Abbreviations}

ADC: Apparent diffusion coefficient; CR: Complete response; DW: Diffusion weighted; HCC: Hepatocellular carcinoma; NR: Not responding; PD: Progressive disease; PR: Partial response; R: Responding; RECIST: Response Evaluation Criteria in Solid Tumors; S: Stable disease; SD: Stable disease

\section{Acknowledgements}

To Prof. Dr. Ihab Samy Reda and Prof. Dr. Ahmed Hafez for their supervision and advise

\section{Authors' contributions}

NE collected the data and consents and performed the analysis of the study. SM revised and assessed the radiological issues of the study, performing the analysis of the study, and the corresponding author of the current paper. SZ contributed to case selection, prescribing the chemotherapy, and follow-up for the medical issues of the patients. All authors read and approved the final manuscript.

\section{Funding}

The author states that this work has not received any funding.

\section{Availability of data and materials}

The datasets used and/or analyzed during the current study are available from the corresponding author on reasonable request.

\section{Ethics approval and consent to participate}

This study was approved by the Ethics Committee of the Faculty of Medicine, Alexandria University on March 16, 2017; reference number of approval 01028116 . All patients included in this study gave written informed consent to participate in this research (All the patients were older than 16 years old).

\section{Consent for publication}

All patients included in this research gave written informed consent to publish the data contained within this study.

\section{Competing interests}

The author of this manuscript declares no relationships with any companies, whose products or services may be related to the subject matter of the article.

\section{Author details}

'Department of Diagnostic and Intervention Radiodiagnosis, Medical Research Institute, Alexandria University, 165 Alhoreia Street, Alexandria 21561, Egypt. ${ }^{2}$ Department of Oncology, Medical Research Institute, Alexandria University, Alexandria, Egypt.

Received: 9 September 2019 Accepted: 21 October 2019

\section{Published online: 16 December 2019}

References

1. Torre LA, Bray F, Siegel RL, Ferlay J, Lortet-Tieulent J, Jemal A (2015) Global cancer statistics, 2012. CA Cancer J Clin 65(2):87-108

2. Ding Y, Rao SX, Meng T, Chen C, Li R, Zeng MS (2014) Preoperative evaluation of colorectal liver metastases: comparison of gadopentetate dimeglumine and gadoxetic-acid-enhanced 1.5-T MRI. Clin Imaging 38(3): 273-278

3. Bai G, Wang Y, Zhu Y et al (2019) Prediction of Early Response to chemotherapy in breast cancer liver metastases by diffusion-weighted MR imaging. Technol Cancer Res Treat 18:1533033819842944

4. Beckers RCJ, Lambregts DMJ, Lahaye MJ et al (2018) Advanced imaging to predict response to chemotherapy in colorectal liver metastases - a systematic review. HPB 20(2):120-127

5. Kim JH, Joo I, Kim TY, Han SW, Kim YJ, Lee JM et al (2016) Diffusion-related MRI parameters for assessing early treatment response of liver metastases to cytotoxic therapy in colorectal cancer. AJR Am J Roentgenol 207(3):W26W32

6. Taouli B, Koh DM (2010) Diffusion-weighted MR imaging of the liver. Radiology 254(1):47-66

7. Charles-Edwards EM, de Souza NM (2006) Diffusion-weighted magnetic resonance imaging and its application to cancer. Cancer Imaging 6:135-143
8. Kele PG, van der Jagt EJ (2010) Diffusion weighted imaging in the liver. World J Gastroenterol 16(13):1567-1576

9. Padhani AR, Liu G, Koh DM, Chenevert TL, Thoeny HC, Takahara T et al (2009) Diffusion-weighted magnetic resonance imaging as a cancer biomarker: consensus and recommendations. Neoplasia 11(2):102-125

10. American Cancer Society (2017) Colorectal cancer facts \& figures 2017-2019. American Cancer Society, Atlanta

11. Schulz W, Hagen C, Hort W (1985) The distribution of liver metastases from colonic cancer. A quantitative postmortem study. Virchows Arch A Pathol Anat Histopathol 406(3):279-284

12. Rockwell S, Dobrucki IT, Kim EY, Marrison ST, Vu VT (2009) Hypoxia and radiation therapy: past history, ongoing research, and future promise. Curr Mol Med 9(4):442-458

13. Vaupel $P$, Mayer A (2007) Hypoxia in cancer: significance and impact on clinical outcome. Cancer Metastasis Rev 26(2):225-239

14. Cui Y, Zhang XP, Sun YS, Tang L, Shen L. Apparent diffusion coefficient: potential imaging biomarker for prediction and early detection of response to chemotherapy in hepatic metastases. (2008). Radiology; 248(3):894-900.

15. Kim SH, Lee JM, Hong SH, Kim GH, Lee JY, Han JK, et al. Locally advanced rectal cancer: added value of diffusion-weighted MR imaging in the evaluation of tumor response to neoadjuvant chemo- and radiation therapy. (2009). Radiology; 253(1):116-125.

16. DeVries AF, Kremser C, Hein PA, Griebel J, Krezcy A, Ofner D et al (2003) Tumor microcirculation and diffusion predict therapy outcome for primary rectal carcinoma. Int J Radiat Oncol Biol Phys 56(4):958-965

17. Harry VN, Semple SI, Gilbert FJ, Parkin DE (2008) Diffusion-weighted magnetic resonance imaging in the early detection of response to chemoradiation in cervical cancer. Gynecol Oncol 111(2):213-220

18. Nilsen L, Fangberget A, Geier O, Olsen DR, Seierstad T (2010) Diffusionweighted magnetic resonance imaging for pretreatment prediction and monitoring of treatment response of patients with locally advanced breast cancer undergoing neoadjuvant chemotherapy. Acta Oncol 49(3):354-360

19. Koh DM, Scurr E, Collins D, Kanber B, Norman A, Leach MO et al (2007) Predicting response of colorectal hepatic metastasis: value of pretreatment apparent diffusion coefficients. AJR Am J Roentgenol 188(4):1001-1008

20. Mardor $Y$, Roth Y, Ochershvilli A, Spiegelmann R, Tichler T, Daniels $D$ et al (2004) Pretreatment prediction of brain tumors' response to radiation therapy using high b-value diffusion-weighted MRI. Neoplasia 6(2):136-142

21. Pickles MD, Gibbs P, Lowry M, Turnbull LW (2006) Diffusion changes precede size reduction in neoadjuvant treatment of breast cancer. Magn Reson Imaging 24(7):843-847

22. Marugami N, Tanaka T, Kitano S, Hirohashi S, Nishiofuku H, Takahashi A et al (2009) Early detection of therapeutic response to hepatic arterial infusion chemotherapy of liver metastases from colorectal cancer using diffusionweighted MR imaging. Cardiovasc Intervent Radiol 32(4):638-646

23. Heijmen L, Verstappen MC, Ter Voert EE, Punt CJ, Oyen WJ, de Geus-Oei LF et al (2012) Tumour response prediction by diffusion-weighted MR imaging: ready for clinical use? Crit Rev Oncol Hematol 83(2):194-207

24. Mungai F, Pasquinelli F, Mazzoni LN, Virgili G, Ragozzino A, Quaia E et al (2014) Diffusion-weighted magnetic resonance imaging in the prediction and assessment of chemotherapy outcome in liver metastases. Radiol Med 119(8):625-633

\section{Publisher's Note}

Springer Nature remains neutral with regard to jurisdictional claims in published maps and institutional affiliations. 